\title{
Anomalous Magnetic Contributions to Hall Effect in $\mathrm{Ho}_{0.5} \mathrm{Lu}_{0.5} \mathrm{~B}_{12}$
}

\author{
V. Krasnorussky ${ }^{a, *}$, A. Bogach $^{a, b}$, N. Sluchanko ${ }^{a, c}, \mathrm{~S}$ DemisheV $^{a, c}$, N. Shitsevalova $^{d}$,

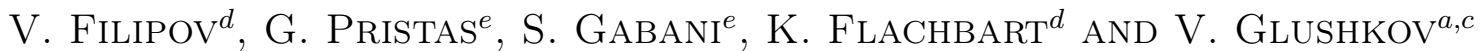 \\ ${ }^{a}$ A.M. Prokhorov General Physics Institute of the Russian Academy of Sciences, \\ 38 Vavilov str., Moscow, 119991 Russia \\ ${ }^{b}$ National University of Science and Technology MISIS, Moscow, 119049 Russia \\ ${ }^{c}$ Moscow Institute of Physics and Technology, 9 Institutsky per., Dolgoprudny, Moscow Region, 141700 Russia \\ ${ }^{d}$ Frantsevich Institute for Problems of Materials Science, NASU, 3 Krzhizhanovsky str., Kyiv, 03142 Ukraine \\ ${ }^{e}$ Institute of Experimental Physics, SAS, 47 Watsonova str., Košice, 04001 Slovakia
}

\begin{abstract}
Strongly correlated electron system $\mathrm{Ho}_{0.5} \mathrm{Lu}_{0.5} \mathrm{~B}_{12}$ possesses a cooperative dynamic Jahn-Teller instability of the boron cage that affects the rattling modes of loosely bound rare-earth ions, a frustrated antiferromagnetic (AF) ground state and dynamic charge stripes. The Hall effect, resistivity, and magnetization were studied on the single crystals of this $\mathrm{AF}$ metal $\left(T_{\mathrm{N}} \approx 3.4 \mathrm{~K}\right)$ at temperatures $1.9-4.2 \mathrm{~K}$ in magnetic field $B \leq 7 \mathrm{~T}$ applied along the principal axis of the fcc lattice. Two components of anomalous Hall effect (AHE) were identified on Hall resistivity $\rho_{\mathrm{H}}(B)$ curves. The first AHE component to be proportional to magnetization is detected both in the $\mathrm{AF}$ and in the paramagnetic state of $\mathrm{Ho}_{0.5} \mathrm{Lu}_{0.5} \mathrm{~B}_{12}$. The second one that contributes to AHE only in the AF phase has the amplitude increasing with lowering of temperature. Both AHE components are strongly anisotropic having maximal amplitudes for $\boldsymbol{B} \|(100)$. The observed AHE features are discussed in terms of spin chirality and Berry phase effects, which could develop in the exotic spin texture of $\mathrm{Ho}_{0.5} \mathrm{Lu}_{0.5} \mathrm{~B}_{12}$ under strong charge carrier scattering on dynamic charge stripes.
\end{abstract}

DOI: 10.12693/APhysPolA.137.767

PACS/topics: anomalous Hall effect, antiferromagnetism, spin chirality

\section{Introduction}

The antiferromagnetic (AF) ground state in $\mathrm{HoB}_{12}$ $\left(T_{\mathrm{N}} \approx 7.4 \mathrm{~K}\right)$ arises under strong spin fluctuations characterized by a pronounced anisotropy of correlation radia and different divergence $\sim T^{-1}$ and $\sim\left(T-T_{\mathrm{N}}\right)^{-1 / 4}$, respectively, along and perpendicular to $\langle 111\rangle$ axis of the fcc lattice [1]. This exotic magnetism was found to be strongly influenced by the cooperative dynamic Jahn-Teller instability of the boron cage [2], that modulates significantly the rattling modes of loosely bound rare-earth ions, and stimulates dynamic charge stripe formation in this AF metal [3]. When considering frustration of $\mathrm{AF}$ exchange in $\mathrm{HoB}_{12}[1]$ it is of great interest also to study the specific features of anomalous Hall effect (AHE), which could be induced by non-coplanar spin textures and related Berry-phase effects [4] in the system with strong spin fluctuations.

To analyze the AHE in both AF and paramagnetic phases of this frustrated magnetic system, the critical temperature and magnetic field of the $\mathrm{AF}$ state of $\mathrm{HoB}_{12}$ were reduced replacing half of $\mathrm{Ho}$ ions with non-magnetic $\mathrm{Lu}$. Here, we present the study of charge transport (resistivity and Hall effect) and magnetization of $\mathrm{Ho}_{0.5} \mathrm{Lu}_{0.5} \mathrm{~B}_{12}$ single crystals measured at low temperatures $(1.9-4.2 \mathrm{~K})$ in magnetic fields up to $7 \mathrm{~T}$.

\footnotetext{
*corresponding author; e-mail: krasnorussky@mail.ru
}

\section{Experimental details}

The crystals were grown by inductive zone melting in argon gas atmosphere and cut from the same ingot as those ones studied earlier in [3]. Charge transport parameters were measured by standard 5 -probe technique with dc current applied along $\langle 110\rangle$ direction. Static magnetization was detected by Quantum Design SQUID magnetometer. All the data were collected for magnetic field applied along $\langle 100\rangle,\langle 110\rangle$, and $\langle 111\rangle$ axis of the fcc lattice in order to study the anisotropy of transport and magnetic properties. The internal magnetic field was corrected by the demagnetization field.

\section{Results and Discussion}

The selected transport characteristics of $\mathrm{Ho}_{0.5} \mathrm{Lu}_{0.5} \mathrm{~B}_{12}$ single crystals are presented in Fig. 1. Lowering of temperature results in a hump detected in the resistivity below Neel temperature $T_{\mathrm{N}} \approx 3.4 \mathrm{~K}$ (see inset in Fig. 1). This feature is specific for AF metals and originates from the conduction electrons scattering induced by the onset of "magnetic" Brillouin zone due to new periodicity of the lattice appearing in the AF state $[5,6]$. At $T=2.1 \mathrm{~K}$ the experimental data allow to resolve clearly the critical magnetic field $B_{\mathrm{N}} \approx 3.4 \mathrm{~T}$, which corresponds to the transition from AF to high-field paramagnetic $(\mathrm{P})$ state of $\mathrm{Ho}_{0.5} \mathrm{Lu}_{0.5} \mathrm{~B}_{12}$ and does not depend on the direction of applied magnetic field (Fig. 1a and 1b). In contrary, magnetoresistance and Hall effect (Fig. 1a and 1b) 


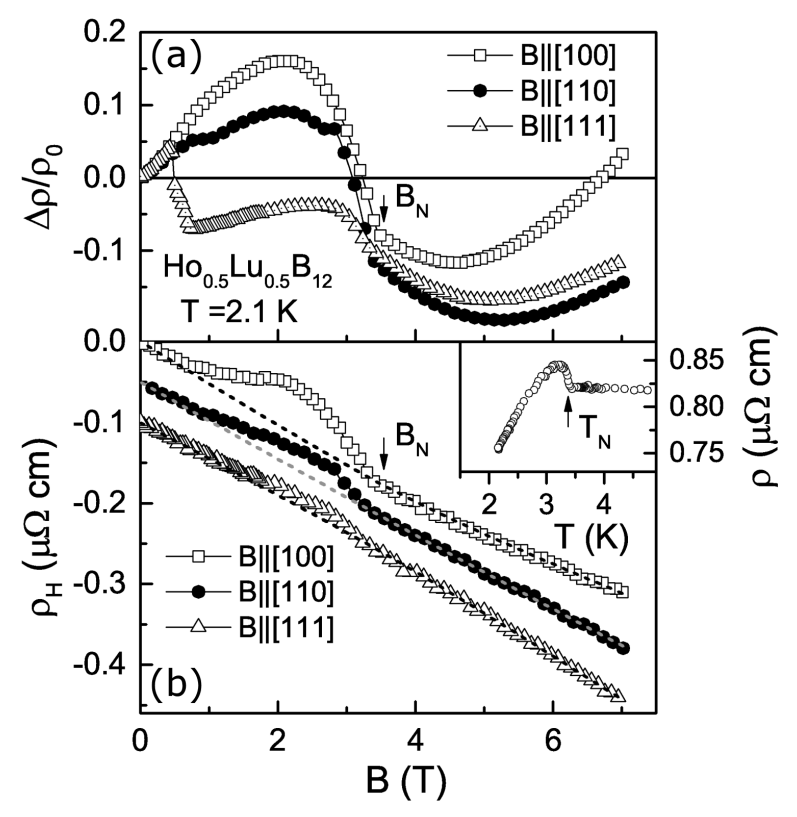

Fig. 1. Magnetoresistance (a), and (b) Hall resistivity of $\mathrm{Ho}_{0.5} \mathrm{Lu}_{0.5} \mathrm{~B}_{12}$ taken at $T=2.1 \mathrm{~K}$ for $\boldsymbol{B} \|[100]$, $\boldsymbol{B} \|[110]$, and $\boldsymbol{B} \|[111]$. For clearance the $\rho_{\mathrm{H}}(B)$ data for $\boldsymbol{B} \|[110]$, and $\boldsymbol{B} \|[111]$ are shifted down by $0.05 \mu \Omega \mathrm{cm}$, and $0.1 \mu \Omega \mathrm{cm}$, respectively. Dash lines are fitting curves for the P phase. Arrows point to the Neel field $B_{\mathrm{N}}$. Inset presents the temperature dependence of resistivity in zero field (Neel temperature $T_{\mathrm{N}} \approx 3.4 \mathrm{~K}$ is shown by the arrow).

in both $\mathrm{AF}$ and $\mathrm{P}$ phases vary with the change of magnetic field direction significantly while the anisotropy of magnetization $M(B)$ (not shown here) does not exceed $2 \%$ for all the temperatures and magnetic fields used in this work. The absence of any sharp features in magnetization related to spin-flip or spin-flop transitions seems to agree with the complicated incommensurate $4 \boldsymbol{q}$ magnetic structure of Ho moments [1]. At the same time magnetoresistance becomes strongly anisotropic in fields $B>B^{*} \approx 0.5 \mathrm{~T}$, so that its amplitude and sign in the AF phase depend on the $B$ direction (Fig. 1a). In $\mathrm{P}$ phase $\left(B>B_{\mathrm{N}}\right)$ the data of $\Delta \rho / \rho_{0}$ can be well described by a sum of positive $\left(\Delta \rho / \rho_{0} \sim B^{2}\right)$ and negative $\left(-\Delta \rho / \rho_{0} \sim M^{2}\right)$ contributions. This, in fact, agrees very well with the previous study [3]. Note also that the anisotropy of $\Delta \rho / \rho_{0}$ in the $\mathrm{P}$ phase appears due to the positive term with the amplitude depending strongly on the $B$ direction. This observation assumes a strong anisotropy of drift mobility with the increasing ratio $\mu_{100} / \mu_{110}$ from 1.3 at $1.9 \mathrm{~K}$, up to 1.5 at $4.2 \mathrm{~K}$.

In turn, the anisotropy of Hall resistivity in the $\mathrm{P}$ phase of $\mathrm{Ho}_{0.5} \mathrm{Lu}_{0.5} \mathrm{~B}_{12}$ is less pronounced (Fig. 1b). The $\rho_{\mathrm{H}}(\boldsymbol{B} \|[100])$ dependence has positive curvature in strong magnetic field, $\rho_{\mathrm{H}}(\boldsymbol{B} \|[110])$ decreases linearly with increase of $B$, and the $\rho_{\mathrm{H}}(\boldsymbol{B} \|[111])$ has negative curvature (Fig. 1b). We used a standard expression to separate the ordinary and anomalous components in Hall effect, namely:

$$
\rho_{\mathrm{H}}=R_{0} B+\rho_{\mathrm{H}}^{A},
$$

where $R_{0}$ is the ordinary Hall coefficient and $\rho_{\mathrm{H}}^{A}$ is the anomalous Hall resistivity. Neither the $\rho_{\mathrm{H}}^{A} \sim \rho^{2} M$ fit, nor the $\rho_{\mathrm{H}}^{A} \sim \rho M$ fit, despite of association with the AHE theory containing the Berry-phase/side-jumping effects and skew scattering, respectively [4], provided a satisfactory fitting to the experimental data in the full range of magnetic fields of the $\mathrm{P}$ region. However, the empirical relation suggested for AHE in ferromagnetic metals $\rho_{\mathrm{H}}^{A}=R_{S} \mu_{0} M\left(R_{S}=\right.$ const $)$, allows to fit the $\rho_{\mathrm{H}}(B)$ data within the experimental accuracy for all the temperatures studied in this work (see dash lines in Fig. 1b).

Parameters $R_{0}$ and $R_{S}$ determined for $\mathrm{Ho}_{0.5} \mathrm{Lu}_{0.5} \mathrm{~B}_{12}$ by linear fitting of the $\rho_{\mathrm{H}} / B=f(M / B)$ plots, are presented in Fig. 2a and Fig. 2b, respectively. Both $R_{0}$ and $R_{S}$ depend strongly on the $\boldsymbol{B}$ direction, and that is a quite unexpected effect for the fcc crystal. The amplitude of the ordinary Hall effect changes twice when comparing the data for $\boldsymbol{B} \|[100]$ and $\boldsymbol{B} \|$ [111] (Fig. 2a). For the same $\boldsymbol{B}$ directions, the AHE component changes its sign from negative (for $\boldsymbol{B} \|[100]$ ) to positive (for $\boldsymbol{B} \|[111]$ ), see Fig. 2b. It is worth noting that no clear features are observed in $R_{0}(T)$ and $R_{S}(T)$ dependencies near $T_{\mathrm{N}}$. This means that $B_{\mathrm{N}}(T)$ is a real AF phase boundary, and the $\mathrm{P}$ phase is uniform below and above $T_{\mathrm{N}}$. Moreover, similar linear trends observed in all $R_{S}(T)$ data at low temperatures (shown by dashed lines in Fig. 2b) may prove the relevance of this AHE component to spin chirality [7-8], which tends to zero due to rising of spin polarization with decreasing of temperature.

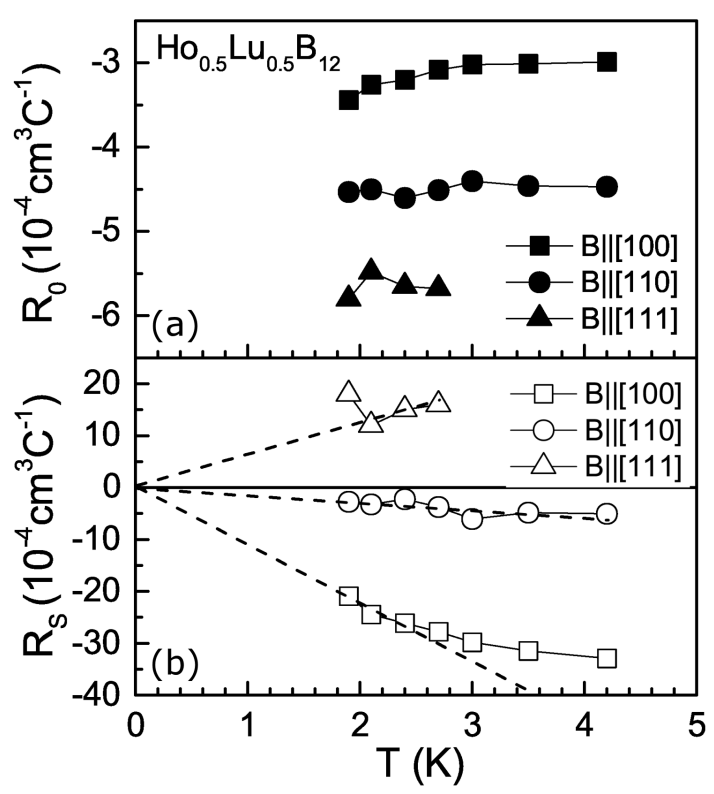

Fig. 2. Temperature dependences of the ordinary (a), and (b) anomalous Hall coefficients for $\mathrm{Ho}_{0.5} \mathrm{Lu}_{0.5} \mathrm{~B}_{12}$ in the paramagnetic state $\left(\boldsymbol{B} \| B_{\mathrm{N}}(T)\right)$ for magnetic field applied along (100), (110), and (111) axis. Dash lines are guides-for-eye. 


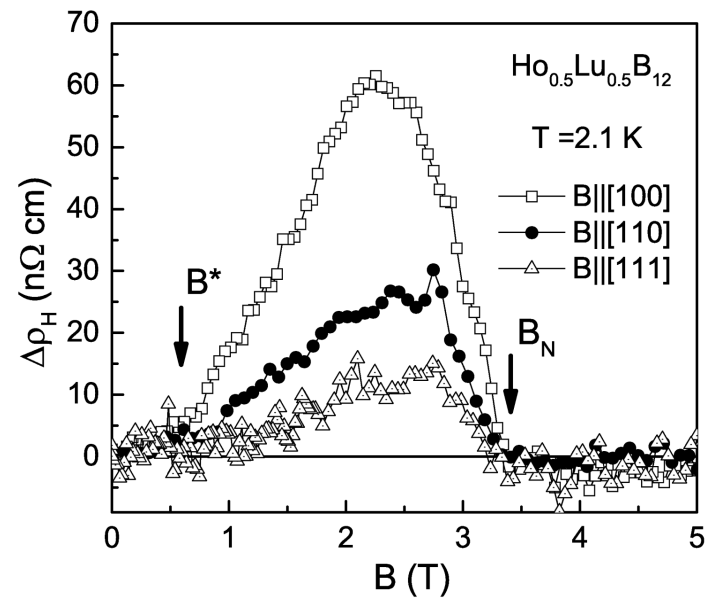

Fig. 3. AHE component $\Delta \rho_{\mathrm{H}}=\rho_{\mathrm{H}}(B)-\left(R_{0} B+\right.$ $\left.R_{S} \mu_{0} M\right)$ in the AF phase of $\mathrm{Ho}_{0.5} \mathrm{Lu}_{0.5} \mathrm{~B}_{12}$ at $T=2.1 \mathrm{~K}$

Actually, such a strong anisotropy of magnetoresistance and Hall effect in the $\mathrm{P}$ phase of $\mathrm{Ho}_{0.5} \mathrm{Lu}_{0.5} \mathrm{~B}_{12}$ (Fig. 1 and 2) may be considered as a manifestation of complex topology of the Fermi surface in this compound [9]. However, the strong negative contribution to magnetoresistance, which is dominant in the studied range of temperatures and magnetic fields (Fig. 1a), and different curvatures of the $\rho_{\mathrm{H}}(\boldsymbol{B} \|[100]), \rho_{\mathrm{H}}(\boldsymbol{B} \|[110])$, and $\rho_{\mathrm{H}}(\boldsymbol{B} \|[111])$ data (Fig. 1b) in the $\mathrm{P}$ phase leave little space to any multi-carrier model with reasonable parameters of charge carriers.

In the $\mathrm{AF}$ phase of $\mathrm{Ho}_{0.5} \mathrm{Lu}_{0.5} \mathrm{~B}_{12}\left(B<B_{\mathrm{N}}(T)\right)$ an additional positive contribution to AHE can be observed in the experimental $\rho_{\mathrm{H}}(B)$ data (see Fig. 1b). The difference $\Delta \rho_{\mathrm{H}}$ between Hall resistivity $\rho_{\mathrm{H}}(B)$ and the fit given by (1) was found to stay positive for all temperatures and magnetic fields used in this work, see Fig. 3. Note that the sign of this anomalous contribution to Hall effect in the AF phase (Fig. 3) is opposite to that of $R_{0}$ (Fig. 2a). The zero AHE contribution in magnetic fields $B<B^{*} \approx 0.5 \mathrm{~T}$ (Fig. 3) may be induced by complicated multiple $\boldsymbol{q}$ order, which averages the extra contribution to Hall effect in low fields. The observable difference in behavior of $\Delta \rho / \rho_{0}$ (Fig. 1a) and $\Delta \rho_{\mathrm{H}}$ (Fig. 3) below $B^{*}$ suggests the different origin of the low field contribution to Hall effect, which is not sensitive to the scattering intensity. The strong dependence of the $\Delta \rho_{\mathrm{H}}$ amplitude on the direction of magnetic field observed under isotropic magnetization may result from the modulation of spin chirality, which is reduced gradually from $\boldsymbol{B} \|[100]$ to $\boldsymbol{B} \|[111]$ (Fig. 3). On the other hand, the largest amplitudes of AHE parameters (i.e., $R_{S} \approx 3 \times 10^{-3} \mathrm{~cm}^{3} / \mathrm{C}$ in Fig. $2 \mathrm{~b}$, and $\Delta \rho_{\mathrm{H}} \approx 60 \mathrm{n} \Omega \mathrm{cm}$ in Fig. 3), as well as the smallest Hall coefficient $\left(\left|R_{0}\right| \approx 3 \times 10^{-4} \mathrm{~cm}^{3} / \mathrm{C}\right.$ in Fig. 2a) observed in the AF and $\mathrm{P}$ phases for $\boldsymbol{B} \|[100]$ may be treated as specific features of charge carrier scattering on dynamic charge stripes, which form a nanometer size filamentary structure of conducting channels in the matrix of $\mathrm{Ho}_{1-x} \mathrm{Lu}_{x} \mathrm{~B}_{12}$ [3]. Further, investigation of $\mathrm{AHE}$ in these compounds with the different Lu content in an extended range of temperatures and magnetic fields is required to reveal the origin of this anomalous behavior.

\section{Conclusion}

Two components of the anomalous Hall effect (AHE) in strongly correlated electron system $\mathrm{Ho}_{0.5} \mathrm{Lu}_{0.5} \mathrm{~B}_{12}$ were identified from $\rho_{\mathrm{H}}(B)$ curves by separating the ordinary contribution to Hall effect. A strong anisotropy of both the anomalous and ordinary Hall resistivity with respect to the magnetic field was observed. The AHE component in the $\mathrm{P}$ phase, which is proportional to magnetization $\left(R_{S} \mu_{0} M\right)$, tends to zero with decreasing temperature. That may be associated with the decrease of spin chirality due to the rise of spin polarization. The anisotropic AHE contribution observed in the AF phase may be induced by spin chirality modulation or by Berry-phase effects, which develop in the exotic magnetic texture of $\mathrm{Ho}_{1-x} \mathrm{Lu}_{x} \mathrm{~B}_{12}$ under strong charge carrier scattering on dynamic charge stripes [3].

\section{Acknowledgments}

This work was supported by the Russian Science Foundation (project 17-12-01426, resistivity and magnetoresistance study), Russian Foundation for Basic Research (project 18-02-01152, Hall effect measurements) and by the Slovak agencies VEGA (project 2/0032/16) and APVV (project 17-0020, resistivity and magnetization data).

\section{References}

[1] K. Siemensmeyer, K. Habicht, Th. Lounkai, S. Mat'aš, S. Gabáni, N. Shitsevalova, E. Wulf, K. Flachbart, J. Low Temp. Phys. 146, 581 (2007).

[2] N. Sluchanko, A. Bogach, N. Bolotina, V. Glushkov, S. Demishev, A. Dudka, V. Krasnorussky, O. Khrykina, K. Krasikov, V. Mironov, V.B. Filipov, N. Shitsevalova, Phys. Rev. B 97, 035150 (2018).

[3] N.E. Sluchanko, A.L. Khoroshilov, A.V. Bogach, V.V. Voronov, V.V. Glushkov, S.V. Demishev, V.N. Krasnorussky, K.M. Krasikov, N.Yu. Shitsevalova, V.B. Filipov, JETP Lett. 107, 30 (2018).

[4] N. Nagaosa, J. Shinova, S. Onoda, A.H. MacDonald, N.P. Ong, Rev. Mod. Phys. 82, 1539 (2010).

[5] G.T. Meaden, Contemp. Phys. 12, 313 (1971).

[6] R.J. Elliott, F.A. Wedgwood, Proc. Phys. Soc. 81, 846 (1963).

[7] Y. Taguchi, Y. Oohara, H. Yoshizawa, N. Nagaosa, Y. Tokura, Science 291, 2573 (2001).

[8] H. Ishizuka, N. Nagaosa, Sci. Adv. 4, eaap9962 (2018).

[9] H. Harima, A. Yanase, T. Kasuya, J. Magn. Magn. Mater. 47-48, 567 (1985). 